\title{
COMPACTNESS AND INCOMPACTNESS PHENOMENA IN SET THEORY
}

\author{
JAMES CUMMINGS
}

\begin{abstract}
We prove two results with a common theme: the tension between compactness and incompactness phenomena in combinatorial set theory. Theorem 1 uses PCF theory to prove a sort of "compactness" for a version of Dzamonja and Shelah's strong non-reflection principle. Theorem 2 investigates Jensen's subcompact cardinals and their relationship with stationary set reflection and the failure of the square principle.
\end{abstract}

\section{INTRODUCTION}

A persistent theme in combinatorial set theory is the tension between compactness and incompactness, or to put it another way between reflection and nonreflection. These are not very precise concepts, so we illustrate with two lists of examples; list one contains ideas and results which (in the view of the author) are instances of compactness, and list two contains some more or less complementary instances of incompactness.

- Compactness:

(1) The compactness theorem for first-order logic

(2) Large cardinals and generic embeddings

(3) Stationary reflection principles

(4) The tree property

(5) Silver's theorem that GCH can't first fail at $\aleph_{\omega_{1}}$

(6) Shelah's singular compactness theorem

- Incompactness:

(1) The failure of compactness for many infinitary logics

(2) The Axiom of Constructibility $V=L$ and its consequences (e.g. square principles)

(3) Non-reflecting stationary sets

(4) Aronszajn trees

(5) Magidor's theorem that GCH can fail first at $\aleph_{\omega}$

(6) Constructions by Shelah and others for "almost free non-free" objects (e.g. groups)

It is our thesis that many interesting problems arise from considering the extent of some form of compactness, or the tension between some compactness principle (for example the existence of large cardinals) and a different incompactness principle (for example the existence of square sequences). This way of thinking is one of the main motivations in our joint work with Foreman and Magidor on singular cardinal combinatorics $[8,7]$ to which this paper is in some sense a sequel.

To describe a favourite problem about the extent of compactness, we recall that a $\kappa$-Aronszajn tree is a tree of height $\kappa$, with every level of size less than $\kappa$ and with 
no cofinal branches. The cardinal $\kappa$ is said to have the tree property if there are no $\kappa$-Aronszajn trees.

It is provable in ZFC that $\aleph_{0}$ has the tree property and $\aleph_{1}$ does not, but by work of Mitchell [19] " $\aleph_{2}$ has the tree property" is independent of ZFC and has the strength of a weakly compact cardinal. Each of the statements " $\aleph_{n}$ has the tree property for all $n$ with $2 \leq n<\omega$ " [6] and " $\aleph_{\omega+1}$ has the tree property" [18] is known to be consistent and of very high consistency strength, but the consistency of their conjunction is open.

Of course there are ideas and results which do not fit nicely into this picture. For example the principle $\nabla_{\kappa}$ seems to be a reflection principle, saying as it does that any subset of $\kappa$ is anticipated by the diamond sequence at many points below $\kappa$; indeed $\rangle_{\kappa}$ follows from the assumption that $\kappa$ is a sufficiently large cardinal. On the other hand $\nabla_{\kappa}$ can be used to construct non-compact objects.

Theorem 1 is an application of PCF theory, which shows that a version of a combinatorial principle called Strong Non-Reflection (SNR) does not fail first at $\aleph_{\omega+1}$. As the name suggests SNR is a principle which would not be out of place in our "Incompactness" list, so we have a sort of compactness for an incompactness property.

Theorem 2 is about the tension between large cardinal axioms and Jensen's square principle. We start by observing that Jensen's quasicompactness principle is sufficiently strong to derive the failure of $\square_{\lambda}$ for $\lambda$ singular. Theorem 2 shows that a similar proof idea from the weaker subcompactness principle is doomed to failure (which is not to say that another proof may not succeed). An exact determination of the consistency strength of the failure of $\square_{\lambda}$ for $\lambda$ singular will presumably have to await progress in the inner model program.

\section{StRong NON-REFLECTION}

The following "Strong Non-Reflection" principle was introduced by Dzamonja and Shelah [12]: here $\kappa$ and $\lambda$ are regular and $\omega<\kappa<\lambda$.

Definition 1. $S N R(\lambda, \kappa)$ holds iff there is a function $f: \lambda \rightarrow \kappa$ such that for all $\alpha \in \lambda \cap \operatorname{cof}(\kappa)$ there is $C \subseteq \alpha$ club with $f \uparrow C$ strictly increasing.

It follows from $S N R(\lambda, \kappa)$ that for every stationary $S \subset \lambda$ there is a stationary $T \subseteq S$ such that $T$ reflects to no point of cofinality $\kappa$ (where $T$ reflects at $\alpha$ if $T \cap \alpha$ is stationary in $\alpha$ ). To see this we just apply Fodor's theorem to find $T$ on which $f$ is constant and argue that this set can not reflect.

Remark 1. Dzamonja and Shelah observed that for any $\kappa$ and $\lambda, S N R(\lambda, \kappa)$ follows from Jensen's global square principle [16]. In particular $\operatorname{SNR}(\lambda, \kappa)$ holds in $L$.

Dzamonja, Shelah and the author [5, 9, 11] studied the SNR principles and used them to prove independence results about reflection. Dzamonja and Shelah made the following definition, which can be seen as a measure of the extent of a certain kind of incompactness in the universe of set theory.

Definition 2. $u(\kappa)$ is the least regular $\lambda>\kappa$ such that $S N R(\lambda, \kappa)$ fails, assuming that such a cardinal exists. If no such cardinal exists then by convention $u(\kappa)=\infty$, where $\lambda<\infty$ for all cardinals $\lambda$. 
Dzamonja and Shelah showed by a hard forcing argument that $u(\kappa)$ can be the successor of a singular cardinal (other kinds of regular cardinal are considerably easier to achieve). We state and prove a result which sheds some light on the difficulties. Theorem 1 is closely related to a theorem from our joint work with Foreman and Magidor [7] which gives a similar kind of compactness for the square principle.

It is convenient to work with a variation on SNR: here $\mu<\kappa<\lambda$ and they are all regular cardinals.

Definition 3. $S N R(\lambda, \kappa, \mu)$ holds iff there exists $f: \lambda \cap \operatorname{cof}(\mu) \rightarrow \kappa$ such that for all $\alpha \in \lambda \cap \operatorname{cof}(\kappa)$ there is $C \subseteq \alpha$ club with $f \uparrow C \cap \operatorname{cof}(\mu)$ strictly increasing.

The principle $S N R(\lambda, \kappa, \mu)$ implies that every stationary subset of $\lambda \cap \operatorname{cof}(\mu)$ contains a stationary set reflecting at no points in $\lambda \cap \operatorname{cof}(\kappa)$. Principles of this type can be used [9] to separate the phenomena of stationary reflection at different cofinalities.

Theorem $1(\mathrm{CH})$. If the principle $S N R\left(\aleph_{n}, \aleph_{2}, \aleph_{1}\right)$ holds for all finite $n>2$, then the principle $S N R\left(\aleph_{\omega+1}, \aleph_{2}, \aleph_{1}\right)$ holds.

Before proving Theorem 1 we need a few PCF-theoretic preliminaries. We note that the hypothesis of $\mathrm{CH}$ in Theorem 1 may not be necessary, and that this is related to an important open question in PCF theory which we discuss below. We refer the reader to the survey papers $[4,1]$ and Shelah's book [20] for background on PCF theory.

For an infinite set $A \subseteq \omega$, we let $\prod_{n \in A} \aleph_{n}$ be the set of functions $f$ such that $\operatorname{dom}(f)=A$ and $f(n) \in \aleph_{n}$ for all $n \in A$. There are various relations on $\prod_{n \in A} \aleph_{n}$ which will concern us. Given $f, g \in \prod_{n \in A} \aleph_{n}$ we say that $f$ is dominated by $g$ $(f<g)$ if and only if $f(n)<g(n)$ for all $n \in A$. We say $f$ is eventually dominated by $g\left(f<<^{*} g\right)$ if and only if $f(n)<g(n)$ for all sufficiently large $n \in A$, and also define $f \leq^{*} g$ if and only if $f(n) \leq g(n)$ for all large $n \in A$. Similarly $f$ is eventually equal to $g\left(f={ }^{*} g\right)$ if and only if $f(n)=g(n)$ for all sufficiently large $n \in A$. Finally $f<^{\infty} g$ if and only if $f(n)<g(n)$ for unboundedly many $n \in A$.

We will use the following theorem by Shelah; apart from our appeal to this basic result, this section of the paper is essentially self-contained.

Fact 1. There is an infinite $A \subseteq \omega$ and a sequence $\left\langle f_{\alpha}: \alpha<\aleph_{\omega+1}\right\rangle$ increasing and cofinal in $\prod_{n \in A} \aleph_{n}$ with the eventual domination ordering.

For the rest of this section we fix $A$ and $\left\langle f_{\alpha}: \alpha<\aleph_{\omega+1}\right\rangle$ as in Fact $1 .^{1}$

If $\beta$ is a limit ordinal less than $\aleph_{\omega+1}$, then we say that a function $g \in \prod_{n \in A} \aleph_{n}$ is an exact upper bound (eub) for $\left\langle f_{\alpha}: \alpha<\beta\right\rangle$ if and only if

(1) For all $\alpha<\beta, f_{\alpha}<^{*} g$.

(2) For all $h \in \prod_{n \in A} \aleph_{n}$, if $h<^{*} g$ then there is $\alpha<\beta$ such that $h<^{*} f_{\alpha}$.

This is equivalent to demanding that $\left\langle f_{\alpha}: \alpha<\beta\right\rangle$ is increasing and cofinal modulo finite in $\prod_{n \in A} g(n)$. It is not difficult to see that an exact upper bound, if it exists, is unique modulo finite.

\footnotetext{
${ }^{1}$ It is interesting to note that there is a canonical maximal choice for $A$ which is well-defined modulo finite, though we will not use this.
} 
We will need a resullt by Shelah guaranteeing many points where an eub exists. The result is an easy corollary ${ }^{2}$ of Shelah's quite technical "trichotomy theorem", but in the interests of making this paper self-contained we sketch a more direct proof (we are really just working through the trichotomy construction and cutting some corners with the help of $\mathrm{CH})$.

Fact 2. Let $C H$ hold. If $\operatorname{cf}(\beta)=\aleph_{2}$ then $\left\langle f_{\alpha}: \alpha<\beta\right\rangle$ has an eub $H$ such that $\operatorname{cf}(H(n))=\aleph_{2}$ for all but finitely many $n$.

Proof. We define a sequence of functions $H_{\zeta} \in \prod_{n \in A} \aleph_{n}$ which are attempts to build a least upper bound (lub), that is to say a function $H$ such that

(1) $f_{\alpha}<^{*} H$ for all $\alpha<\beta$.

(2) There is no $\bar{H} \leq^{*} H$ such that $\bar{H}<^{\infty} H$ and $f_{\alpha}<^{*} \bar{H}$ for all $\alpha<\beta$.

Our construction will be such that if $\zeta<\eta$ then $H_{\eta} \leq^{*} H_{\zeta}$ and $H_{\eta}<^{\infty} H_{\zeta}$. The construction will proceed for at most $\aleph_{2}$ many steps.

$H_{0}=f_{\beta}$. If $H_{\zeta}$ is an lub then we stop the construction, otherwise we choose $H_{\zeta+1} \leq^{*} H_{\zeta}$ such that $H_{\zeta+1}<^{\infty} H_{\zeta}$ and $f_{\alpha}<^{*} H_{\zeta+1}$ for all $\alpha<\beta$. At limit $\lambda<\aleph_{2}$ we work as follows: let $X_{n}=\left\{H_{\zeta}(n): \zeta<\lambda\right\}$ and for every $\gamma<\beta$ let $G_{\gamma}(n)=\min \left(X_{n} \backslash f_{\gamma}(n)\right)$, or zero if $X_{n} \subseteq f_{\gamma}(n)$.

It is routine to check that $f_{\alpha}<{ }^{*} G_{\gamma}$ for all $\alpha<\beta$, that $G_{\gamma} \leq{ }^{*} H_{\zeta}$ for all $\zeta<\lambda$ and that $\gamma<\delta \Longrightarrow G_{\gamma} \leq^{*} G_{\delta}$. By $\mathrm{CH}$ there are only $\aleph_{1}^{\aleph_{0}}=\aleph_{1}$ possibilities for the $=^{*}$-equivalence class of $G_{\gamma}$, and since $\operatorname{cf}(\beta)=\aleph_{2}$ that equivalence class must stabilise: we choose $H_{\lambda}$ so that $H_{\lambda}={ }^{*} G_{\gamma}$ for all large $\gamma<\beta$.

We claim that the constuction of the $H_{\zeta}$ must halt before $\aleph_{2}$ steps. Otherwise we may define a function $F$ from $\left[\aleph_{2}\right]^{2}$ to $\omega$, by $F(\zeta, \eta)=n$ for $n$ minimal with $H_{\eta}(n)<H_{\zeta}(n)$. The Erdos-Rado theorem then gives a decreasing $\aleph_{1}$-sequence of ordinals, which is impossible.

We have constructed an lub $H$. We claim it is an eub. To see this let $g<^{*} H$ and suppose for a contradiction that $Y_{\gamma}=\left\{n: g(n)>f_{\gamma}(n)\right\}$ is infinite for all $\gamma$. Clearly $\gamma<\delta$ implies that $Y_{\delta}$ is contained in $Y_{\gamma} \bmod$ finite, and so by $\mathrm{CH}$ again there is a fixed $Z$ such that $Y_{\gamma}$ is equal to $Z \bmod$ finite for all large $\gamma<\beta$. Define $\bar{H}$ by $\bar{H}(n)=H(n)$ for $n \notin Z, \bar{H}(n)=g(n)$ for $n \in Z$; clearly $\bar{H} \leq^{*} H, \bar{H}<^{\infty} H$ and $f_{\alpha}<{ }^{*} \bar{H}$ for all $\alpha$, contradiction!

To finish we show that $\operatorname{cf}(H(n))=\aleph_{2}$ for all but finitely many $n$. Suppose first that $\operatorname{cf}(H(n))<\aleph_{2}$ for every $n$ in some infinite subset $B$ of $A$, and fix $A_{n} \subseteq$ $H(n)$ cofinal with ot $\left(A_{n}\right)=\operatorname{cf}(H(n))$ for every $n \in B$. Now by $\mathrm{CH} \prod_{n \in B} A_{n}$ has cardinality $\aleph_{1}$, so we may find $f_{\alpha}$ such that for every $f \in \prod_{n \in B} A_{n}$ we have $f<^{*} f_{\alpha} \uparrow B$. Since $f_{\alpha}<^{*} H$ we may find $g \in \prod_{n \in B}$ such that $f_{\alpha} \uparrow B<^{*} g$, which is a contradiction.

Now suppose that $\operatorname{cf}(H(n))>\aleph_{2}$ for every $n$ in some infinite subset $B$ of $A$. Let $\left\langle\beta_{i}: i<\aleph_{2}\right\rangle$ be increasing and cofinal in $\beta$ and define $f \in \prod_{n \in B} H(n)$, by setting $f(n)=\sup _{i} f_{\beta_{i}}(n)$ for all $n \in B$. Then since $H$ is an eub and the $\beta_{i}$ are cofinal we may find $i$ such that $f<^{*} f_{\beta_{i}} \uparrow B$, which is a contradiction.

Fact 2 is our only use of $\mathrm{CH}$. As we discuss further at the end of this section, it is unclear whether the assumption of $\mathrm{CH}$ can be removed from Fact 2 or indeed from

\footnotetext{
${ }^{2}$ For the experts: we just observe that points of cofinality greater than the continuum can not fall into the Bad or Ugly cases of the trichotomy.
} 
Theorem 1. Next we characterise those points where an eub of uniform uncountable cofinality exists. Again this result is due to Shelah.

Fact 3. The following are equivalent for $\beta$ of uncountable cofinality.

(1) There exists $g$ an eub for $\left\langle f_{\alpha}: \alpha<\beta\right\rangle$ with $\operatorname{cf}(g(n))=\operatorname{cf}(\beta)$ for all but finitely many $n$.

(2) There exists $g$ an eub for $\left\langle f_{\alpha}: \alpha<\beta\right\rangle$ and an uncountable regular $\lambda$ with $\operatorname{cf}(g(n))=\lambda$ for all but finitely many $n$.

(3) There exists a sequence of functions $\left\langle h_{\eta}: \eta<\operatorname{cf} \beta\right\rangle$ in ${ }^{A} O N$ which is pointwise increasing and is cofinally interleaved with $\left\langle f_{\alpha}: \alpha<\beta\right\rangle$ in the eventual domination ordering (which is to say that each function in each of the sequences is eventually dominated by some function from the other sequence).

(4) For every $Y \subseteq \beta$ which is unbounded in $\beta$ there is $Z \subseteq Y$ unbounded in $\beta$ and $n<\omega$, such that $\operatorname{ot}(Z)=\operatorname{cf}(\beta)$ and $\left\langle f_{\alpha}(m): \alpha \in Z\right\rangle$ is strictly increasing for $m \in A$ with $m>n$.

Proof. It is immediate that 1 implies 2. Given 2, define a pointwise increasing sequence of functions $\left\langle h_{\eta}: \eta<\lambda\right\rangle$ such that $\left\langle h_{\eta}(n): \eta<\lambda\right\rangle$ is increasing and cofinal in $g(n)$ for all but finitely many $n$. Since $h_{\eta}<^{*} g, h_{\eta}<^{*} f_{\alpha}$ for some $\alpha<\beta$ : conversely if $\alpha<\beta$ then $f_{\alpha}<^{*} g$, and since $\lambda$ is uncountable and the $h_{\eta}$ are pointwise increasing we may choose $\eta$ such that $f_{\alpha}<^{*} h_{\eta}$. It follows that $\lambda=\operatorname{cf}(\beta)$ so 2 implies 3.

Given 3, let $Y \subseteq \beta$ be unbounded. We may clearly choose $\eta_{j}<\operatorname{cf}(\beta)$ and $\zeta_{j} \in Y$ such that $g_{\eta_{j}}<^{*} f_{\zeta_{j}}<^{*} g_{\eta_{j+1}}$ for $j<\operatorname{cf}(\beta)$, and then choose $n_{j} \in A$ such that $g_{\eta_{j}}(m)<f_{\zeta_{j}}(m)<g_{\eta_{j+1}}(m)$ for $m>n_{j}$. Since $\operatorname{cf}(\beta)$ is uncountable we may find $T \subseteq \operatorname{cf}(\beta)$ unbounded and $n$ such that $n_{j}=n$ for all $j \in T$, and then let $Z=\left\{\zeta_{j}: j \in T\right\}$. If $j_{1}<j_{2}$ are in $T$ and $m>n$ then $f_{\zeta_{j_{1}}}(m)<g_{\eta_{j_{1}+1}}(m) \leq$ $g_{\eta_{j_{2}}}(m)<f_{\zeta_{j_{2}}}(m)$, so 3 implies 4 .

Given 4 we let $Y=\beta$, choose a suitable $Z$ and $n$ and define $H$ by $H(m)=$ $\sup _{\alpha \in Z} f_{\alpha}(m)$. Clearly $\operatorname{cf}(H(m))=\operatorname{cf}(\beta)$ for all but finitely many $m$, and $f_{\alpha}<^{*} H$ for all $H$. Now if $f<^{*} H$, then since $\operatorname{cf}(\beta)$ is uncountable and $\left\langle f_{\alpha}(m): \alpha \in Z\right\rangle$ is strictly increasing for $m \in A$ with $m>n$ we may choose $\alpha \in Z$ such that $f<^{*} f_{\alpha}$. It follows that $H$ is an eub so 4 implies 1 .

Remark 2. In the course of this proof, we saw that for any cofinally interleaved sequence $\left\langle h_{\eta}: \eta<\operatorname{cf} \beta\right\rangle$ as in 3, the pointwise supremum of $\left\langle h_{\eta}: \eta<\operatorname{cf} \beta\right\rangle$ is an exact upper bound for $\left\langle f_{\alpha}: \alpha<\beta\right\rangle$.

Let $G$ be the set of all those $\beta<\aleph_{\omega+1}$ of uncountable cofinality such that there is an eub $g$ for $\left\langle f_{\alpha}: \alpha<\beta\right\rangle$ with $\operatorname{cf}(g(n))=\operatorname{cf}(\beta)$ for all but finitely many $n$. We claim that if $\gamma \in G$ there is a club subset $C$ of $\gamma$ such that all points of $C$ with uncountable cofinality are in $G$. To see this we use " 1 implies 3 " from Fact 3 to construct $\left\langle h_{\eta}: \eta<\operatorname{cf}(\gamma)\right\rangle$ which is cofinally interleaved with $\left\langle f_{\alpha}: \alpha<\gamma\right\rangle$, and then let $C$ be the set of $\delta<\gamma$ such that $\left\langle f_{\alpha}: \alpha<\delta\right\rangle$ is cofinally interleaved with $\left\langle h_{\eta}: \eta<\bar{\eta}\right\rangle$ for some $\bar{\eta}<\eta ; C$ is clearly club, and by "3 implies 1" from Fact 3 every element of $C$ with uncountable cofinality is in $G$.

The claim of the last paragraph and the fact that under $\mathrm{CH}$ every point of cofinality $\aleph_{2}$ is in $G$ are the keys to the proof of Theorem 1 . We will use the set $G$ as a scaffolding on which to build a function witnessing $S N R\left(\aleph_{\omega+1}, \aleph_{2}, \aleph_{1}\right)$. 
Before starting the proof of Theorem 1, it will be convenient to make some cosmetic adjustments to $A$ and the sequence $\left\langle f_{\alpha}: \alpha<\aleph_{\omega+1}\right\rangle$. It follows easily from the discussion above that we may assume that

(1) The minimum element of $A$ is at least 3.

(2) The sequence $\left\langle f_{\alpha}: \alpha<\aleph_{\omega+1}\right\rangle$ is continuous, that is to say that whenever an exact upper bound for an initial segment $\left\langle f_{\alpha}: \alpha<\beta\right\rangle$ exists then $f_{\beta}$ is an exact upper bound.

(3) If $\beta \in G$ and $\operatorname{cf}(\beta) \leq \aleph_{2}$ then $\operatorname{cf}\left(f_{\beta}(n)\right)=\operatorname{cf}(\beta)$ for all $n \in A$.

Proof of Theorem 1: We assume that CH holds. We fix $F_{n}: \aleph_{n} \cap \operatorname{cof}\left(\aleph_{1}\right) \rightarrow \aleph_{2}$ witnessing $S N R\left(\aleph_{n}, \aleph_{2}, \aleph_{1}\right)$ for $2<n<\omega$. We define $F$ for $\gamma \in G \cap \operatorname{cof}\left(\aleph_{1}\right)$ by $F(\gamma)=\sup _{n} F_{n}\left(f_{\gamma}(n)\right)$ and verify that $F$ is a witness for $S N R\left(\aleph_{\omega+1}, \aleph_{2}, \aleph_{1}\right)$.

We fix a point $\delta<\aleph_{\omega+1}$ of cofinality $\aleph_{2}$. Since CH holds Fact 2 tells us that $\delta \in$ $G$, so that by our cosmetic work above $f_{\delta}$ is an exact upper bound for $\left\langle f_{\alpha}: \alpha<\delta\right\rangle$ and $\operatorname{cf}\left(f_{\delta}(n)\right)=\aleph_{2}$ for all $n \in A$. We fix for each $n$ a club $C_{n}$ in $f_{\delta}(n)$ such that $F_{n}$ is increasing on $C_{n} \cap \operatorname{cof}\left(\aleph_{1}\right)$, and $C_{n}$ has order type $\aleph_{2}$. Then we define $h_{\eta}$ for $\eta<\aleph_{2}$ by setting $h_{\eta}(n)$ to be the $\eta^{\text {th }}$ point in $C_{n}$; by the argument for " 2 implies 3 " in Fact 3, $\left\langle h_{\eta}: \eta<\aleph_{2}\right\rangle$ is pointwise increasing and is cofinally interleaved with $\left\langle f_{\alpha}: \alpha<\delta\right\rangle$.

We may now fix $E$ club in $\delta$ with order type $\aleph_{2}$, such that for every point $\gamma \in E \cap \operatorname{cof}\left(\aleph_{1}\right)$ there is a (necessarily unique) $\zeta(\gamma) \in \aleph_{2} \cap \operatorname{cof}\left(\aleph_{1}\right)$ such that the sequences $\left\langle f_{\alpha}: \alpha<\gamma\right\rangle$ and $\left\langle h_{\eta}: \eta\langle\zeta(\gamma)\rangle\right.$ are cofinally interleaved. Thinning out $E$ if necessary we may arrange that ot $\left(\zeta(\gamma) \cap \operatorname{cof}\left(\aleph_{1}\right)\right)=\zeta(\gamma)$ for all $\gamma \in E \cap \operatorname{cof}\left(\aleph_{1}\right)$. Fixing for the moment some $\gamma \in E \cap \operatorname{cof}\left(\aleph_{1}\right)$, it follows from the discussion above that the pointwise supremum of $\left\langle h_{\eta}: \eta\langle\zeta(\gamma)\rangle\right.$ is an exact upper bound for $\left\langle f_{\alpha}: \alpha<\gamma\right\rangle$, so by continuity and the fact that the sets $C_{n}$ are closed we see that $f_{\gamma}={ }^{*} h_{\zeta(\gamma)}$.

Since $F_{n}$ is strictly increasing on $C_{n} \cap \operatorname{cof}\left(\aleph_{1}\right), F_{n}\left(h_{\eta}(n)\right) \geq \operatorname{ot}\left(\eta \cap \operatorname{cof}\left(\aleph_{1}\right)\right)$ for all $n$ and $\eta$, so in particular $F_{n}\left(f_{\gamma}(n)\right)=F_{n}\left(h_{\zeta(\gamma)}(n)\right) \geq \zeta(\gamma)$ for all large $n$. By the definition of $F$ it follows that $F(\gamma) \geq \zeta(\gamma)$ for all $\gamma \in E \cap \operatorname{cof}\left(\aleph_{1}\right)$. Clearly $\zeta(\gamma)$ is strictly increasing with $\gamma$, and so we may thin out $E$ to a club subset $E^{*}$ of $\delta$ with the property that $\zeta\left(\gamma_{1}\right)>F\left(\gamma_{0}\right)$ for all $\gamma_{1} \in E^{*} \cap \operatorname{cof}\left(\aleph_{1}\right)$ and $\gamma_{0} \in E^{*} \cap \gamma_{1} \cap \operatorname{cof}\left(\aleph_{1}\right)$.

If $\gamma_{0}$ and $\gamma_{1}$ are points of $E^{*} \cap \operatorname{cof}\left(\aleph_{1}\right)$ with $\gamma_{0}<\gamma_{1}$ then $F\left(\gamma_{0}\right)<\zeta\left(\gamma_{1}\right) \leq$ $F\left(\gamma_{1}\right)$. We have verified that $F$ witnesses $\operatorname{SNR}\left(\aleph_{\omega+1}, \aleph_{2}, \aleph_{1}\right)$ and this concludes the proof.

The set $G$ (the set of "good points") turns out to be an interesting invariant of the universe of set theory; for more about this point of view see the papers [8] and [7] and To whet the reader's appetite we note that

- Modulo the club filter, $G$ is independent of the choice of the scale $\left\langle f_{\alpha}: \alpha<\right.$ $\left.\aleph_{\omega+1}\right\rangle$.

- Jensen's weak square principle $\square_{\aleph_{\omega}}^{*}$ implies that almost all points of uncountable cofinality are good.

- Starting from very large cardinals it is known $[14,7]$ that we may build models in which there are stationarily many ungood points of cofinality $\aleph_{1}$. In particular this statement follows from Martin's Maximum (which implies the negation of $\mathrm{CH}$ ) and also from the strong Chang conjecture $\left(\aleph_{\omega+1}, \aleph_{\omega}\right) \rightarrow\left(\aleph_{1}, \aleph_{0}\right)$ (which is consistent with $\left.\mathrm{CH}\right)$, so is consistent both with $\mathrm{CH}$ and its negation. 
- It is open whether or not there may be stationarily many ungood points of cofinality $\aleph_{2}$. It is known that any point of cofinality greater than $2^{\aleph_{0}}$ is good.

\section{QUASICOMPACT AND SUBCOMPACT CARDINALS}

Jensen showed [16] that $\square_{\lambda}$ holds in $L$ for all $\lambda$, and also [10] that if $0^{\sharp}$ does not exist then $L$ computes correctly the successors of $V$-singular cardinals; it follows that if $0^{\sharp}$ does not exist then $\square_{\lambda}$ holds for all singular $\lambda$, and so combinatorial statements which are incompatible with $\square_{\lambda}$ (e.g. the non-existence of a $\lambda^{+}$-Aronszajn tree) must have a substantial consistency strength.

Workers in the inner model program have constructed $L$-like models (the socalled " $L[\vec{E}]$-models") which can contain substantial large cardinals. We refer the reader to the survey paper $[21]$ for more details. The $L[\vec{E}]$-models are only known to exist up to a certain point in the large cardinal hierarchy (roughly a measurable limit of Woodin cardinals). It is anticipated they will be shown to exist at higher levels of the large cardinal hierarchy, going past the subcompact and quasicompact cardinals defined below; modulo the assumption of existence it is already possible to analyse the fine structure of these hypothetical models.

A natural problem is to prove that $\square_{\lambda}$ holds in $L[\vec{E}]$-models, but there are limits on what can be done in this direction. Jensen has identified a large cardinal property called subcompactness (qv) and has shown that if $\kappa$ is subcompact then $\square_{\kappa}$ fails. Schimmerling and Zeman have closed the case in $L[\vec{E}]$-models, by showing that in such models $\square_{\lambda}$ holds exactly when $\lambda$ is not subcompact.

We recall that $H_{\lambda}$ is the set of those $X$ such that the transitive closure of $\{X\}$ has cardinality less than $\lambda$. Informally it is often helpful to think of $H_{\lambda}$ as the set of those $X$ which can be coded by bounded subsets of $\lambda$.

Definition 4 (Jensen). Let $\kappa$ be a cardinal.

(1) $\kappa$ is quasicompact iff for all $A \subseteq H_{\kappa^{+}}$there are $\lambda>\kappa, \pi: H_{\kappa^{+}} \rightarrow H_{\lambda^{+}}$ and $B \subseteq H_{\lambda^{+}}$such that $\pi$ is an elementary embedding from $\left(H_{\kappa^{+}}, \in, A\right)$ to $\left(H_{\lambda^{+}}, \in, B\right), \pi(\kappa)=\lambda$ and the critical point of $\pi$ is $\kappa$.

(2) $\kappa$ is subcompact iff for all $A \subseteq H_{\kappa^{+}}$there are $\beta<\kappa, \pi: H_{\beta^{+}} \rightarrow H_{\kappa^{+}}$ and $b \subseteq H_{\beta^{+}}$such that $\pi$ is an elementary embedding from $\left(H_{\beta^{+}}, \in, b\right)$ to $\left(H_{\kappa^{+}}, \in, A\right), \pi(\beta)=\kappa$ and the critical point of $\pi$ is $\beta$.

Remark 3. The quasicompactness of $\kappa$ is witnessed by the existence of a family of superstrong extenders with critical point $\kappa$, and similarly the subcompactness of $\kappa$ is witnessed by the existence of a family of superstrong extenders with target $\kappa$.

If $\kappa$ is quasicompact then $\kappa$ is measurable and subcompact, and the least subcompact cardinal is not measurable.

Theorem 2 is motivated by the problem of calibrating the large cardinal strength needed to make $\square_{\lambda}$ fail for $\lambda$ singular. Before stating and proving Theorem 2 we note that a quasicompact cardinal will suffice for this.

Fact 4 (Foreman and Magidor [8]). Let $\kappa$ be measurable and let $S=\kappa^{+} \cap \operatorname{cof}(<\kappa)$. Assume that every stationary subset of $S$ reflects at a point of cofinality less then $\kappa$; then the same reflection property holds in any generic extension by Prikry forcing.

Fact 5 (Jensen [15]). If $\kappa$ is quasicompact then stationary subsets of $\kappa^{+} \cap \operatorname{cof}(<\kappa)$ reflect at some point of cofinality less than $\kappa$. 
Sketch of the proof of Fact 5: Suppose for a contradiction that $T \subseteq \kappa^{+} \cap \operatorname{cof}(<\kappa)$ is stationary and non-reflecting, and let $\pi:\left(H_{\kappa^{+}}, \in, T\right) \rightarrow\left(H_{\lambda^{+}}, \in, U\right)$ be as in the definition of quasicompactness. $U$ may not be stationary (stationarity is not first-order) but every initial segment is non-stationary, and so we can choose $C$ disjoint from $\pi$ " $T$. Pulling back $C$ we get $D$ club in $\kappa^{+}$with $\pi$ " $D \cap \operatorname{cof}(<\kappa) \subseteq C$, so $D \cap T=\emptyset$. Contradiction!

Since $\square_{\kappa}$ implies that every stationary subset of $\kappa^{+}$has a non-reflecting stationary subset, it follows that doing Prikry forcing at a quasicompact cardinal $\kappa$ gives a model where $\kappa$ is singular and $\square_{\kappa}$ fails. The following result shows that the same scenario can not be made to work starting with a measurable subcompact cardinal.

We will use the idea of strategic closure [13] for a poset $\mathbb{P}$. Consider a game in which two players I and II collaborate to build a decreasing sequence of conditions $p_{i}$ in $\mathbb{P}$, with player I playing $p_{i}$ for $i$ odd and player II playing $p_{i}$ for $i$ even; player II loses a run of the game when a position is reached in which she can not move. If $\kappa$ is an uncountable regular cardinal and for every $\delta<\kappa$ II has a strategy which enables her to play for $\delta$ moves, then $\mathbb{P}$ adds no sequences of length less than $\kappa$.

Theorem 2. It is consistent (modulo the existence of a supercompact cardinal) that there exists $\kappa$ which is measurable and subcompact, and every stationary subset of $\kappa^{+}$contains a non-reflecting stationary subset.

Sketch of the proof of Theorem 2: We use arguments similar to those of Apter and Shelah's papers [2,3]. Let $\kappa$ be a Laver indestructible [17] supercompact cardinal, that is to say $\kappa$ remains supercompact in any extension by $\kappa$-directed closed forcing. Let GCH hold at and above $\kappa$.

We define a poset $\mathbb{P}_{0}$ for adding a non-reflecting stationary set in $\kappa^{+}$. Conditions are functions $f$ such that $\operatorname{dom}(f)<\kappa^{+}, \operatorname{rge}(f) \subseteq\{0,1\}$ and for every $\delta \leq \operatorname{dom}(f)$ of uncountable cofinality there is a club subset $C$ of $\delta$ such that $f \uparrow C$ is constant with value zero. The ordering is extension.

It is easy to see that in the strategic closure game player II can keep going for $\kappa^{+}$moves by extending I's play by a single zero at every successor step, and taking unions at limit steps. In the next paragraph we will check this in some detail. In particular $\mathbb{P}_{0}$ adds no $\kappa$-sequences.

We claim that $\mathbb{P}_{0}$ adds the characteristic function of a set which is stationary in every cofinality up to $\kappa$. To see this let $\delta \leq \kappa$ be regular and let $\dot{C}$ be a $\mathbb{P}_{0}$ name for a club subset of $\kappa^{+}$. Consider a run of the strategic closure game of length $\delta+1$ where $f_{i}$ is played at stage $i$. Player I plays so that for every even $i<\delta, f_{i+1}$ forces that $\dot{C} \cap\left(\operatorname{dom}\left(f_{i}\right), \operatorname{dom}\left(f_{i+1}\right)\right) \neq \emptyset$. For odd $i<\delta$ player II lets $f_{i+1}=f_{i} \cup\left\{\left(\operatorname{dom}\left(f_{i}\right), 0\right)\right\}$. For limit $i$ she lets $\gamma_{i}=\sup _{j<i} \operatorname{dom}\left(f_{j}\right)$ and then sets $f_{i}=\bigcup_{j<i} f_{j} \cup\left\{\left(\gamma_{i}, 0\right)\right\}$ if $i<\delta, f_{i}=\bigcup_{j<i} f_{j} \cup\left\{\left(\gamma_{i}, 1\right)\right\}$ if $i=\delta$. The key point is that for every limit $i$ player II has arranged that $f_{i}$ is zero on a club set in $\gamma_{i}$, thereby guaranteeing that $f_{i}$ is a condition. It is routine to check that $f_{\delta}$ is a condition and that $f_{\delta}$ forces that $\gamma_{\delta}$ is in $\dot{C}$.

In fact the stationary subset added by $\mathbb{P}_{0}$ has stationary intersection with every stationary subset of $\kappa^{+}$from the ground model. To see this fix $T \in V$ a stationary subset of $\kappa^{+}$and $\dot{C}$ a name for a club. Build a run of length $\kappa^{+}$of the strategic closure game, where I plays as in the last paragraph and II adjoins a single zero to the play so far at each of her turns. With the same notation as in the last paragraph 
the $\gamma_{i}$ form a club subset of $\kappa^{+}$, and so we may find $i$ limit with $\gamma_{i} \in T$. Then the function $f_{i} \cup\left\{\left(\gamma_{i}, 1\right)\right\}$ is a condition forcing that $\gamma_{i}$ is in $\dot{C}$.

We now define in $V^{\mathbb{P}_{0}}$ a poset $\mathbb{Q}_{0}(S)$ to destroy the stationarity of the set $S$ added by $\mathbb{P}_{0}$. Conditions are closed bounded subsets disjoint from $S$, ordered by end-extension. It is easy to check that $\mathbb{P}_{0} * \mathbb{Q}_{0}$ has a dense $\kappa^{+}$-closed subset, consisting of those $(f, \check{c})$ such that $\max (c)+1=\operatorname{dom}(f)$ and $f\lceil c$ is constant with value zero. With more work it can be shown that $\mathbb{P}_{0} * \mathbb{Q}_{0}$ is equivalent to the standard poset for adding a Cohen subset of $\kappa^{+}$.

Let $\mathbb{P}$ be the product of $\kappa^{++}$copies of $\mathbb{P}_{0}$ with supports of size $\kappa$, and let $S_{i}$ be the stationary set added by the $i^{\text {th }}$ copy. Let $\mathbb{Q}$ be the product of $\mathbb{Q}_{0}\left(S_{i}\right)$ for $i<\kappa^{++}$, again with supports of size $\kappa$. As in the case of $\mathbb{P}_{0}$ and $\mathbb{Q}_{0}$, it can be checked that all the sets $S_{i}$ are stationary in $V^{\mathbb{P}}$ and that $\mathbb{P} * \mathbb{Q}$ has a dense $\kappa^{+}$-closed subset (and is in fact equivalent to the poset for adding $\kappa^{++}$Cohen subsets of $\kappa^{+}$). The usual $\Delta$-system argument shows that $\mathbb{P}$ is $\kappa^{++}$-c.c.

Let $V_{1}=V[G]$ for some $\mathbb{P}$-generic $G$, and let $V_{2}=V_{1}[H]$ for some $\mathbb{Q}$-generic $H$. $V_{1}$ is the model we want, $V_{2}$ is used in the proof that $\kappa$ is subcompact in $V_{1}$. The following claims are immediate.

- The power set of $\kappa$ is the same in each of $V, V_{1}$ and $V_{2}$. It follows that all these models compute $H_{\kappa^{+}}$in the same way, and also that $\kappa$ is measurable in $V_{1}$.

- By the assumption of indestructibility, $\kappa$ is supercompact in $V_{2}$.

We claim that in $V_{1}$ every stationary subset of $\kappa^{+}$contains a non-reflecting stationary subset. Let $S \in V_{1}$ be such a stationary set. The forcing $\mathbb{P}$ is $\kappa^{++}$c.c. and so $S$ is determined by the first $\beta$ coordinates in $\mathbb{P}$ for some $\beta<\kappa^{++}$; an argument like that given above for $\mathbb{P}_{0}$ now shows that if $T$ is the stationary set added by copy $\beta$ of $T$ then $S \cap T$ is stationary in $V^{\mathbb{P}}$.

We need to check that $\kappa$ is subcompact in $V_{1}$. Let $A$ be a predicate on $H_{\kappa^{+}}$with $A \in V_{1}$. Let $j: V_{2} \rightarrow M_{2}$ be an embedding in $V_{2}$ witnessing $\kappa$ is $\kappa^{+}$-supercompact. Then in $V_{2}$ the map $j\left\lceil H_{\kappa^{+}}\right.$is elementary from $\left(H_{\kappa^{+}}, \in, A\right)$ to $\left(H_{j(\kappa)^{+}}^{M_{2}}, \in, j(A)\right)$. What is more the map $j\left\lceil H_{\kappa^{+}}\right.$lies in $M_{2}$, and $H_{\kappa^{+}}=H_{\kappa^{+}}^{M_{2}}$.

By reflection there are in $V_{2}$ an ordinal $\beta<\kappa$, a predicate $b$ on $H_{\beta^{+}}$and an elementary $\pi$ from $\left(H_{\beta^{+}}, \in, b\right)$ to $\left(H_{\kappa^{+}}, \in, A\right)$. This map $\pi$ lies in $V_{1}$, since it is a subset of $H_{\kappa^{+}}$with cardinality less than $\kappa$, and similarly $H_{\beta^{+}}^{V_{2}}=H_{\beta^{+}}^{V_{1}}$ and $b \in V_{1}$. It follows that $\kappa$ is quasicompact in $V_{1}$.

We conclude with a list of problems:

(1) Can the methods of Theorem 1 be used to "step up" some other combinatorial principles, for example the existence of a non-reflecting stationary set?

(2) What is the largest $L[\vec{E}]$ model such that non-reflecting stationary sets exist (or are dense) in every successor cardinal?

(3) Does a subcompact cardinal (or a measurable subcompact cardinal) suffice to force failure of $\square_{\lambda}$ for $\lambda$ singular?

(4) Do quasicompact cardinals suffice for any more of the notable applications of supercompactness?

(5) In particular, is a quasicompact cardinal sufficient to produce a model where $\square_{\lambda}^{*}$ fails for $\lambda$ singular? 
I would like to thank Mirna Dzamonja, Matt Foreman, Ronald Jensen, Menachem Magidor and Ernest Schimmerling for several helpful conversations. I would also like to thank the anonymous referee for their careful reading of the first version of this paper.

Remark 4. Matt Foreman has pointed out that if player II has a strategy which allows her to play for $\kappa^{+}$moves in the strategic closure game on a poset $\mathbb{P}$, and $2^{\kappa}=\kappa^{+}$, then $\mathbb{P}$ preserves the subcompactness of $\kappa$. So the conclusion of Theorem 2 can be obtained more cheaply by applying the same forcing construction to a measurable subcompact $\kappa$ with $2^{\kappa}=\kappa^{+}$.

Remark 5. Martin Zeman has some preliminary results indicating that, for example, in the $L[\vec{E}]$-models there is a non-reflecting stationary set in $\kappa^{+}$for $\kappa$ the least subcompact cardinal.

\section{REFERENCES}

[1] U. Abraham and M. Magidor. Cardinal arithmetic. To appear in the Handbook of Set Theory.

[2] A. Apter and S. Shelah. Menas' result is best possible. Transactions of the American Mathematical Society, 349(5):2007-2034, 1997.

[3] A. Apter and S. Shelah. On the strong equality between supercompactness and strong compactness. Transactions of the American Mathematical Society, 349(1):103-128, 1997.

[4] M. Burke and M. Magidor. Shelah's pcf theory and its applications. Annals of Pure and Applied Logic, 50:207-254, 1990.

[5] J. Cummings, M. Džamonja, and S. Shelah. A consistency result on weak reflection. Fund. Math., 148(1):91-100, 1995.

[6] J. Cummings and M. Foreman. The tree property. Advances in Mathematics, 133(1):1-32, 1998.

[7] J. Cummings, M. Foreman, and M. Magidor. Tightness. To appear.

[8] J. Cummings, M. Foreman, and M. Magidor. Squares, scales and stationary reflection. Journal of Mathematical Logic, 1(1):35-99, 2001.

[9] J. Cummings and S. Shelah. Some independence results on reflection. J. London Math. Soc. (2), 59(1):37-49, 1999.

[10] K. J. Devlin and R. B. Jensen. Marginalia to a theorem of Silver. In Logic Colloquium, Kiel, 1974, pages 115-142, Berlin, 1975. Springer-Verlag.

[11] M. Džamonja and S. Shelah. Weak reflection at successors of singulars. To appear.

[12] M. Džamonja and S. Shelah. Saturated filters at successors of singular, weak reflection and yet another weak club principle. Annals of Pure and Applied Logic, 79(3):289-316, 1996.

[13] M. Foreman. Games played on Boolean algebras. Journal of Symbolic Logic, 48:714-723, 1983.

[14] M. Foreman and M. Magidor. A very weak square principle. Journal of Symbolic Logic, 62(1):175-196, 1997.

[15] R. Jensen. Circulated notes.

[16] R. B. Jensen. The fine structure of the constructible hierarchy. Annals of Mathematical Logic, 4:229-308, 1972.

[17] R. Laver. Making the supercompactness of $\kappa$ indestructible under $\kappa$-directed closed forcing. Israel Journal of Mathematics, 29:385-388, 1978.

[18] M. Magidor and S. Shelah. The tree property at successors of singular cardinals. Archive for Mathematical Logic, 35(5-6):385-404, 1996.

[19] W. J. Mitchell. Aronszajn trees and the independence of the transfer property. Annals of Mathematical Logic, 5:21-46, 1972.

[20] S. Shelah. Cardinal Arithmetic. Oxford University Press, Oxford, 1994.

[21] J. Steel. An outline of inner model theory. To appear in the Handbook of Set Theory. 